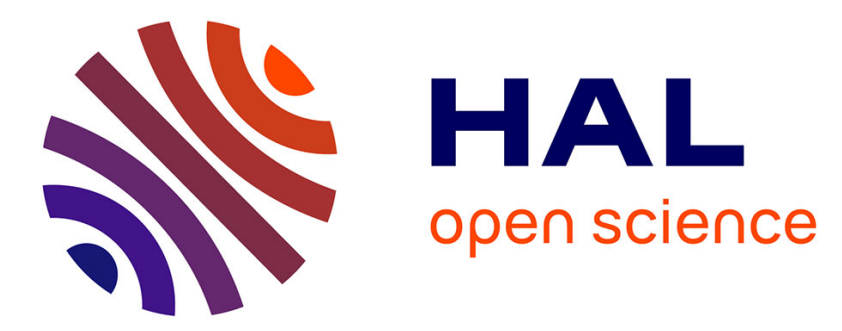

\title{
Impact of Clustering Stability on the Improvement of Time Synchronization in VANETs
}

Khedidja Medani, Makhlouf Aliouat, Zibouda Aliouat

\section{To cite this version:}

Khedidja Medani, Makhlouf Aliouat, Zibouda Aliouat. Impact of Clustering Stability on the Improvement of Time Synchronization in VANETs. 6th IFIP International Conference on Computational Intelligence and Its Applications (CIIA), May 2018, Oran, Algeria. pp.472-483, 10.1007/978-3-31989743-1_41. hal-01913919

\author{
HAL Id: hal-01913919 \\ https://hal.inria.fr/hal-01913919
}

Submitted on 7 Nov 2018

HAL is a multi-disciplinary open access archive for the deposit and dissemination of scientific research documents, whether they are published or not. The documents may come from teaching and research institutions in France or abroad, or from public or private research centers.
L'archive ouverte pluridisciplinaire HAL, est destinée au dépôt et à la diffusion de documents scientifiques de niveau recherche, publiés ou non, émanant des établissements d'enseignement et de recherche français ou étrangers, des laboratoires publics ou privés. 


\title{
Impact of Clustering Stability on the Improvement of Time Synchronization in VANETs
}

\author{
Khedidja MEDANI ${ }^{1,2}$, Makhlouf ALIOUAT ${ }^{1,2}$, and Zibouda ALIOUAT $^{1,2}$ \\ 1 Department of Computer Science, Faculty of sciences, Ferhat Abbas University, \\ Setif, Algeria \\ ${ }^{2}$ LRSD laboratory, Faculty of Science, Computer science department, UFAS1 \\ khadidja_medani@univ-setif.dz maliouat@univ-setif.dz \\ zaliouat@univ-setif.dz
}

\begin{abstract}
Vehicular Ad Hoc NETworks (VANETs) has been developed to be, as a part of the Internet of Vehicles (IoVs), one of the most promoting technologies in the near future. Extensive researches are focusing on developing appropriate solutions in order to provide better Quality of Services (QoS) to these latter. This paper deals with network performance improvement based on clustering mechanism to reduce the number of communications caused by the high frequency of arrival/departure of vehicles while maintaining relevant clock synchronization solutions adequately operating. Thus, we attempt to adopt certain known clustering algorithms to our previous solution of time synchronization in VANETs namely OTRB (Offsets Table Robust Broadcasting) in order to evaluate the impact of a hierarchical communication on time synchronization process. An analytical study with a comparison on the influence of the clustering mechanism is given. The comparison takes place using simulation software NS2 (Network Simulator 2) and VanetMobiSim (VANET Mobility Simulator). The performance parameters include the average of arrival/departure of nodes and the number of isolated nodes. Simulation results reveal that an appropriate method should reduce the overhead of re-clustering and lead to an efficient network coverage with the best time synchronization rate.
\end{abstract}

Keywords: Intelligent transportation systems, VANETs, Clustering, Time synchronization performance.

\section{Introduction}

The emergent Vehicular ad hoc networks (VANETs) tend to improve road safety and users infotainment during displacement. To this end, nodes in VANETs, as moving vehicles, exchange their information using wireless ad hoc communications. Due to the nature of the medium access, vehicles communicate directly with one another or with the available infrastructure using single hop communication. To reach a further destination, multi-hop communications can be 
established using relay nodes. However, the constraints created by the intrinsic characteristic of VANETs, such as the high density of nodes, make data routing a challenging task. To handle such a limitation, data should be spread among an optimized route to ensure reliable delivery with an optimized communication overhead. This can take place by splitting the network into small groups, clusters. For each cluster, a node is elected as a coordinator to communicate data with its members on the one hand and to spread the information to other clusters on the other hand. In addition, several issues depend on a strong assumption of reliable clustering mechanism.

The standard IEEE $802.11 \mathrm{p}$ has been proposed to deal with VANETs safety application. However, due to the high density of nodes, the standard suffers predictability, fairness, low throughput and high collision rate [1]. In addition, the high rate of transmitted data, such as safety packet dissemination, make network prone to face high congestion. So, collisions, especially when large transmission scope is used, lead to packet loss rate increases. To deal with these drawbacks and to improve reliability, many researchers suggest network partitioning into small clusters in order to allow better shared medium access control. Where clustering is used to limit the channel contention and provide fair channel access. Furthermore, clustering algorithms allow power control and packet delivery enhancement.

Time synchronization in VANETs is crucial. Several proposed algorithms, whatever derived from MANETs or not, rely on some sort of logical clustering view [2]. The aim is to provide local time synchronization among the neighboring nodes in order to reach global time synchronization. For instance, in HCS [3] and OTRB [2] protocols, nodes synchronize their clocks by the aid of a randomly selected node, which is considered as a reliable source of time. The time synchronization solution performances are related to the selected node. This elected node can be seen as the coordinator of its neighbors. Such protocols rely on the assumption that the algorithm provides reliable clustering with a minimized number of in / out moving vehicles.

In order to enhance its performances, the present paper carries a study case on the influence of clustering algorithms in time synchronization using OTRB protocol. OTRB algorithm requires the organized clusters to be stable for a period of time enough to achieve the time synchronization. The stability parameters, which refer to the average lifetime of the cluster, is related to the synchronization round period in OTRB. In turn, this latter is related to the synchronized group scale and the desired time precision [2]. Different clustering algorithms in the literature take only into account the distance between nodes and their directions as parameters to form the clusters. However, vehicles displacement is related to the road infrastructure. Even vehicles move roughly with same velocities starting from a nearby zone, they can be found in further ways after a while. Therefore, other parameters, like the velocity of nodes, should be considered in order to deal with the high rate of arrival/departure of nodes.

The main contribution of this paper is to explore the impact of clustering on time synchronization process. To this end, we adopt an appropriate clustering 
algorithm to our previous solution OTRB. The best clustering algorithm should improve the synchronization rate and enhance the communication overhead.

The rest of the paper is organized as follow: section II introduces the main clustering algorithm concepts and their requirement in VNAETs, while section III gives an overview of the proposed solution of clustering issue. Section IV is dedicated to the OTRB protocol. In section V, a comparison of the implemented clustering protocols under OTRB context is discussed in order to evaluate its performance. Finally, section VI concludes the paper.

\section{Basic clustering notions and requirements}

The clustering is defined by the grouping of similar object items into clusters. In [4], a mathematical definition is given as follows: let $X \in R^{m n}$ a set of items representing a set of $m$ objects $x_{i}$ in $R^{n}$. The goal of the clustering algorithm is to divide $X$ into $K$ groups $C_{k}$. Each one is called a cluster. Where all objects that belong to the same cluster are more alike than objects in different clusters. The result of the algorithm is an injective mapping $X \rightarrow C$ of object items $X_{i}$ to clusters $C_{k}$.

The clustering can be exclusive, overlapping or fuzzy. In case of exclusive clustering, one object (node) can be found in one and just one cluster. When, in overlapping clustering, the node can belong to more than one cluster $(>=1)$. Whereas, in fuzzy clustering, for each node is assigned a membership weight. Therefore, the node belongs to the cluster according to its associated weight. Fuzzy algorithms can be used to improve exclusive clustering by avoiding the arbitrary node assignment. In the case, the node will be assigned to the cluster within its membership weight is highest.

In VANETs, clustering is the act of grouping the vehicles into multiple clusters (groups of vehicles). In which, vehicles belonging to the same cluster have the same properties, such as velocity, position and direction.

Under each cluster structure, there is one node acting as a coordinator of the group. It is the cluster-head node $(\mathrm{CH})$. The remaining nodes are classified to cluster-member (CM) and/or cluster-gateway (GW) nodes. In term of communication, a cluster-gateway node is able to communicate with all the clusters in which it belongs. Consequently, this latter represents the relay node of its clusters. In other words, it is the relationship between the neighboring clusters.

It is announced in [5] that "The clusters should reflect some mechanism at work in the domain from which instances or data points are drawn, a mechanism that causes some instances to bear a stronger resemblance to one another than they do to the remaining instances." Regarding to vehicular environments, several parameters have been considered, such as node density, position, speed and direction. These parameters define the requirement that should be employed by any clustering algorithm. From which, we define the following that affects clustering efficiency and network performance [6] [7] :

1. Cluster transmission overhead: The cluster transmission overhead refers as to the average number of packets exchanged to maintain the cluster structure. 
The more the transmission overhead is less, the more the clustering algorithm is desired.

2. Cluster convergence: Cluster Convergence refers as to the time needed for all the nodes in order to join a cluster. The clustering algorithm that takes large convergence time is less suitable.

3. Cluster connect time: this parameter refers as to the rate of connection of the vehicle to one cluster. The highest connect time rate is given, the more suitable clustering algorithm.

4. Cluster stability: the cluster stability refers as to the average lifetime of the cluster. The best clustering algorithm goes after high stability.

\section{Related work}

For the purpose of supporting Quality of Service (QoS) in VANETs networking, stable clustering of the moving vehicles is required. The design of clustering algorithms enables organized medium access control and simplified data communication task. Several works in MANETs literature focus on improving the generated communication overhead by minimizing the number of clusters.

In [8], Gerla et al propose to select the node having the highest number of neighbors as a $\mathrm{CH}$ in its vicinity. The proposed scheme minimizes the number of clusters. On the other hand, it deteriorates the network throughput due to the high rate of CMs within a cluster. This technique is not suitable for VANETs environment characterized by the high density of its nodes.

Other works, such as [9] selects the node with the lowest ID in its vicinity to be a $\mathrm{CH}$ of non-overlapping cluster. This protocol does not take nodes' mobility character into consideration. Then, $\mathrm{CH}$ selection process has to be frequently invoked especially in case of highly networks topology change, such as in VANETs. This will increase the cluster transmission overhead, which in turn reduces the network performances.

$\mathrm{Su}$ and Zhang [10] propose their algorithm which is a time and size based $\mathrm{CH}$ selection. The node that succeeds on sending its invite-to-join packet first and has more neighboring nodes is selected to be a $\mathrm{CH}$. As the vehicles move in and out of the cluster frequently, there is high frequent of arrival and departure of nodes. Thus, maintaining the cluster stability becomes a hard task. Whereas, the mobility of vehicle in VANET systems is a major challenge to ensure clustering stability. Many researches in the current literature attempt to deal such a limitation.

In [11] the authors adopted the idea of [12]. In which, a node will announce itself as a $\mathrm{CH}$ in case it does not receive any Hello packet from its vicinity. Nevertheless, the node is allowed to be a CM in at least one cluster. The authors in the presented algorithm permit the merging of two adjacent clusters if their $\mathrm{CHs}$ come in direct communication range. The new $\mathrm{CH}$ is the one with the suitable weighed factor. This factor is calculated for each $\mathrm{CH}$ considering the mobility, connectivity and distance factors. However, CMs can be found out of the new elected $\mathrm{CH}$, thus the possibility of frequent disconnection between the communicating nodes. 
Kayis and Lichuan in [13] a speed-based classification in order to partition the vehicles into clusters. In which, seven groups of velocities are defined based on the min and the max speed boundaries. The "First Declaration Wins Rule" is adopted to select the set of the CHs. However, the nodes speed changes from node to node and can also change over the time. Therefore, nodes can frequently leave their group to join the more suitable. The algorithm may exhibit a frequent change in the network topology due to high cluster change rate. In addition, the $\mathrm{CH}$ can easily lose the connection with its member nodes.

A heuristic position-based clustering algorithm is proposed in [14]. The cluster formation is obtained by the partition of the network based on the geographical position and the priority associated with each vehicle. Each node calculates its priority value using its node ID, the current time and the eligibility value. The eligibility value increases with the travel time of the node and decreases when the node speed deviates largely from the average speed. The node with the highest priority in all its one-hop neighbors and one of its two-hop neighbors is elected to be a $\mathrm{CH}$. Also, in the proposed technique, in order to optimize the cluster size, a maximum distance between the $\mathrm{CH}$ and its members is predefined. However, simulation results show that the formation of small cluster size increases the cluster reconfiguration rate. On the other hand, large communication range decreases the cluster transmission efficiency.

Wu et al [15] have proposed the Type-based Cluster-forming Algorithm (TCA) in order to reduce the update frequency of CHs in emergency ad hoc networks. Each node is assigned a stability factor, $\mathrm{S}$, which is updated frequently. The node having a lower value of $\mathrm{S}$ has low mobility and reliable connectivity. The node with the lower stability factor is more likely to be elected as a $\mathrm{CH}$. However, because of the assumption that the nodes have slow speeds, this algorithm generates more communication overhead for the cluster updating. This makes the algorithm not suitable to be implemented in VANETs environment.

A distributed $\mathrm{CH}$ selection is proposed in [16] to dynamically organize the network into clusters. The novel proposed algorithm is speed and distance based. The $\mathrm{CH}$ is selected according to its relative speed and the distance within its neighbors. Also, fuzzy logic inference system is used for predicting the future speed and position of all the CMs. As the maintenance phase is adaptable to the drivers' behavior, the proposed algorithm is highly stable. However, the distributed cluster overhead causes the number of messages received/transmitted to be decreased [6]. Also, the algorithm is implemented under medium vehicle density and low-speed conditions.

Rawashdeh and Mahmud [17] attempts to deal with the clustering issue with stability on highway VANET environments. The authors apart from the assumption that "the existence of VANETs nodes in the same geographic proximity does not mean that they exhibit the same mobility pattern", and try to improve the stability by making the network topology less dynamic. In addition to the velocity and the position, the proposed approach takes the location and the velocity difference between the nodes into consideration. Each node runs the algorithm in fully distributed manner. First, a separation of the vehicles into highly mobile 
and low mobile groups is made. The network partition should provide a minimized number of clusters and ensure that all the CMs are stable with respect to one another. Then, the set of nodes with the highest suitable value are elected as $\mathrm{CH}$ nodes. Each node calculates its suitable value, which is calculated based to the velocity and the distance with regard to its stable neighbors. The closer velocity to the average velocities of its stable neighbors the node has, the highest suitable value is given. Simulation results show that the proposed algorithm improves the average cluster lifetime, which increases the stability of network topology.

In [18] a new clustering algorithm in vehicular ad hoc networks is presented. The proposed scheme, called Hierarchical Clustering Algorithm (HCA), provides network two-hop clustering in fully distributed randomized fashion. Three roles can be defined to form the network hierarchy, in which a node can be a cluster head $(\mathrm{CH})$, cluster relay $(\mathrm{CR})$ or slave node. The $\mathrm{CR}$ nodes relay the transmitted data between the $\mathrm{CHs}$ and the slaves. The algorithm forms the clusters running four steps; cluster Relays Selection, ClusterHead Selection, Cluster Formation and Scheduling and Cluster Maintenance. Where it debates fast clusters construction in the first third steps and leave the mobility handling to the maintenance phase. However, the algorithm improves the clustering stability at the price of transmission efficiency and the average of clustering overhead. Also, the high rate of inter-cluster interferences causes frequent cluster changes and message loss due to message collisions. Another clustering algorithm for VANETs is proposed by Hassan abadi et al. in [19]. The authors have presented the Affinity PROpagation for VEhiclar networks (APROVE) protocol which attempts to produce high stability clustering taking into account the mobility parameter of nodes. In the proposed protocol, the $\mathrm{CH}$ selection is based on nodes' interdistance. The closest node to its neighbors is selected. This scheme suffers long cluster convergence time in order to make all the nodes belong to a cluster.

In [20], the authors rely on neighbor vehicles' mobility to maintain VANETs stable clustering in rounds. In which, the node with the lowest neighbor vehicles mobility is elected to be the $\mathrm{CH}$ in its vicinity. Each node is assumed to have a unique identity, ID and maintain a neighbor vehicles table, which includes, for each neighbor, its ID and the neighbor vehicles mobility value. The neighbor vehicle table will be updated at each round and the neighbor vehicle mobility is calculated considering the number of vehicles entering/leaving the service convergence of the node.

In [21] a new oriented VANETs clustering scheme for urban city scenario is presented. In order to improve clustering stability, the proposed scheme considers the vehicles direction, position and the link lifetime estimation. Each node is able to estimate its speed and distance relative to its one-hop neighbors. The nearest vehicle into the central geographical position is likely chosen as a $\mathrm{CH}$. The algorithm defines a safe distance threshold, $D$ which $D$ is less than the transmission range. The vehicles within this value are considered having more stable links with the $\mathrm{CH}$. Therefore, the $\mathrm{CMs}$ are selected by the $\mathrm{CH}$ from its 
one-hop neighbors, in which the cluster size is defined by $L \leq 2 D$.

An analytical study that evaluates the impact of the clustering mechanism on the QoS of VANET systems implementation must be carried out. By way of illustration, we analyze the influence of the clustering protocol performed while maintaining proper clock synchronization using OTRB protocol [2]. The analytical study is carried out using network simulator (NS2) to compare the impact of the rate of arrival/departure nodes on the performance of OTRB protocol. To this end, the following sections demonstrate the behavior of OTRB protocol to maintain synchronized clocks in VANET systems. Then the simulation results are presented.

\section{OTRB protocol overview}

For the purpose of time synchronization in distributed VANET environments, the OTRB protocol is proposed in [2] by Medani et al. The main idea is to set a number of nodes, named transporter nodes, to spread the time information over the entire network. For each transporter node, the time information broadcasted consists of the offsets related to all its neighbors. However, the transporter node is supposed to be a reliable source of time.

The OTRB protocol performs time synchronization relying on pair-wise time synchronization mechanism [22]. Initially, each transporter node broadcasts a synchronization packet to its neighbors. Upon receiving this synchronization packet, neighboring nodes respond by their time values as shown in Figure 1. Then, the transporter node calculates the clock offset relative to each neighbor $i$ and broadcasts the estimated values, as a time table, allowing all of them to synchronize to one another.

The transporter node selection phase has an important impact on OTRB performance in terms of time synchronization rate and communication overhead. Thus, the transporter node should have a higher stability relative to its neighbors. That is, a node leaving its synchronized group before the synchronization will be achieved, generates more synchronization requests. In this case, time synchronization maintenance requires more communication overhead. The more the overhead is generated, the more the communication collisions. In turn, a high rate of collisions decreases the time synchronization rate. On the other hand, the transporter node selection phase should be as fast as possible in order to deal with the time synchronization requirements, delay latency for example.

It is stated in [2] that each transporter node with its synchronized group can be seen as a cluster. Where the transporter node represents the $\mathrm{CH}$ and its neighbors represent the CMs (see figure 2). Each node can join more than one transporter node. In the rest of this paper, we refer to the transporter node as the $\mathrm{CH}$, and to the synchronized group as the CMs.

To show the influence of the clustering algorithm on OTRB performance, in the next sections, we present the simulation and the comparison of three 


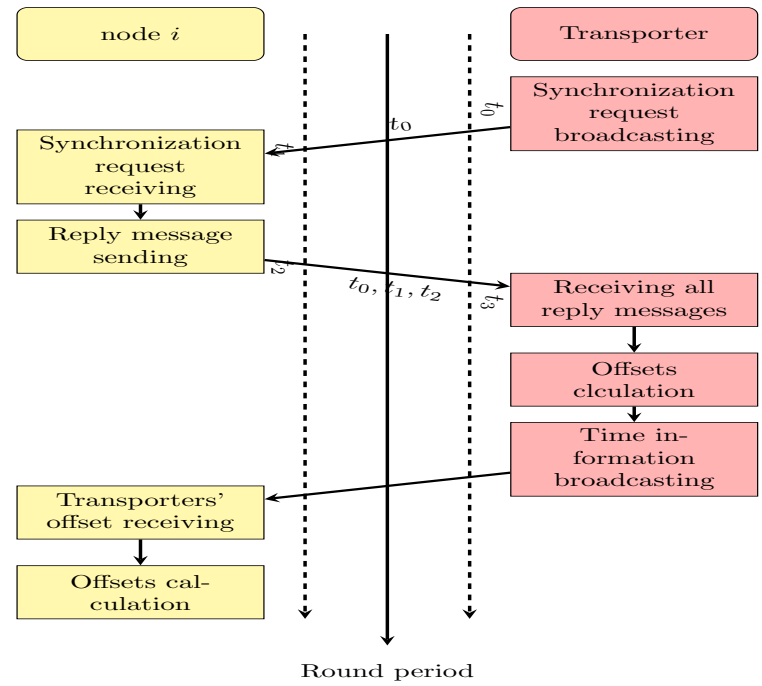

Fig. 1: OTRB protocol process [2].

clustering algorithms (CBLR [12], LID [9] and NMCS [20]) working under OTRB protocol.

\section{Simulation results}

The proposed time synchronization OTRB protocol relies on a strong assumption of stable clustering mechanism. To evaluate its performance and show its better quality of functioning, we adopt three clustering protocols CBLR [12], LID [9] and NMCS [20]to work under the referred OTRB and the behavior of the protocol is observed for each one. The time synchronization rate and the average communication overhead needed to accomplish the synchronization process are measured with regard to the essential clustering stability parameters. In order to meet OTRB requirements, the implementation of these protocols is adapted to maintain overlapping clustering; where a node is permitted to be a $\mathrm{CM}$ in more than one cluster.

1. CBLR: CBLR is originally adopted on OTRB protocol upon its creation. A set of $\mathrm{CHs}$ are selected randomly, in which, a node will announce itself as a $\mathrm{CH}$ if this latter does not receive any synchronization packet from its vicinity. When receiving several synchronization packets, a node is allowed to be CMs in more than one cluster.

2. LID: LID protocol selects nodes with the lowest ID in their vicinity as CHs of non-overlapping clusters. As OTRB protocol requires overlapping clusters, LID is thus modified to allow nodes joining several clusters, if possible, in our contribution. 


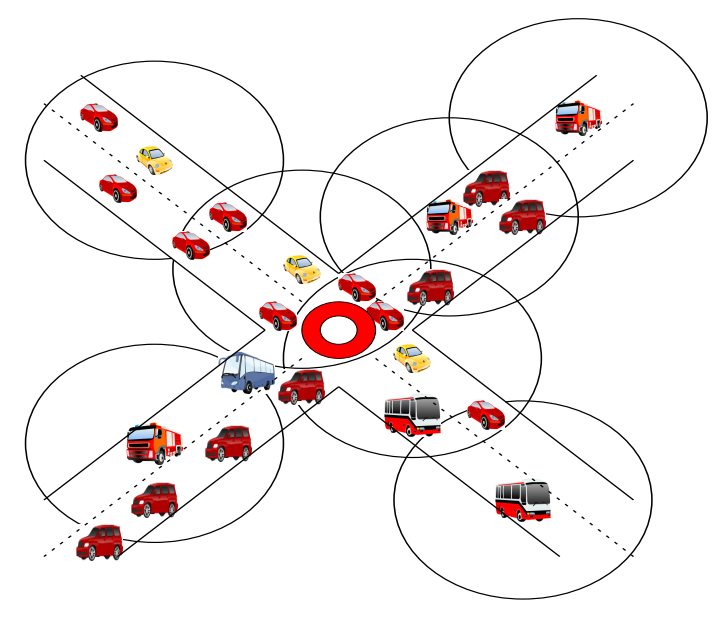

Fig. 2: Network architecture in OTRB [2].

3. NMCS: In NMCS, the node having the lowest neighbor vehicles mobility is selected to be the $\mathrm{CH}$ in its vicinity. NMCS protocol allows overlapping clustering. For each node, a degree is defined as the sum of the number of nodes that have left/arrived the node's transmission range. The sum is then divided by the total number of neighbors to normalize the degree of node's mobility regarding its vicinity. The node having the lowest value for this metric is supposed to be located in a relatively stable environment, indicating that such node is a good candidate for the $\mathrm{CH}$ role.

The simulation of the three referred protocols holds under the parameters shown in Table 1. A scenario of 199 nodes with random positions is initially generated. The mobility model follows the Intelligent Driver Model with Lane Changing (IDM_LC) of VANET Mobility Simulator (VANETMobiSim), in which, vehicles regulate their velocity according to neighboring vehicle movements. This model also supports smart intersection and lane changing management.

Table 1: Simulation parameters

\begin{tabular}{|c|c|c|c|}
\hline & Nodes number & Data transmission range $(m)$ & Time of simulation ( $s$ ) Physical channel \\
\hline
\end{tabular}

In order to evaluate OTRB performances, we observe the changes in the synchronization rate and the communication overhead for each clustering protocol implemented under OTRB. On the other hand, to shed the light on the influence of the clustering protocol stability on OTRB performance, the average of arrival/departure and isolated nodes are captured in parallel. 
As shown in Figure 3, NMCS protocol reduces the average of the nodes moving into new clusters during the synchronization process. That is, NMCS protocol favors nodes located in a stable environment relative to their vicinities to be $\mathrm{CH}$ nodes. In contrast, CBLR and LID protocols do not take into account nodes' mobility while performing the clustering operation. In addition, relying on the lowest ID and the random metrics to select the $\mathrm{CH}$ nodes in LID and CBLR respectively multiplies the risk of having so much number of $\mathrm{CHs}$ and a high number of isolated nodes (see Figure 4).

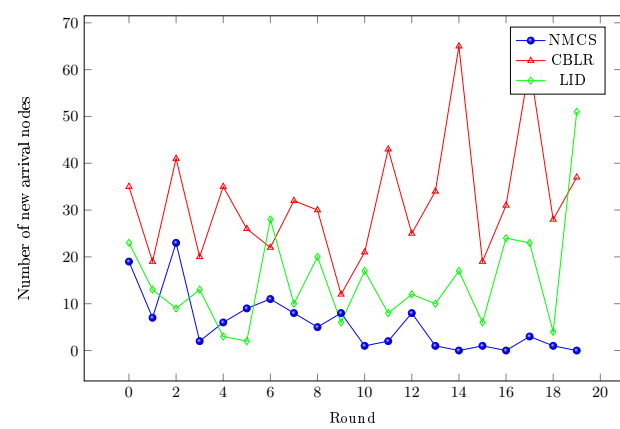

Fig. 3: Average of new arrival nodes per round.

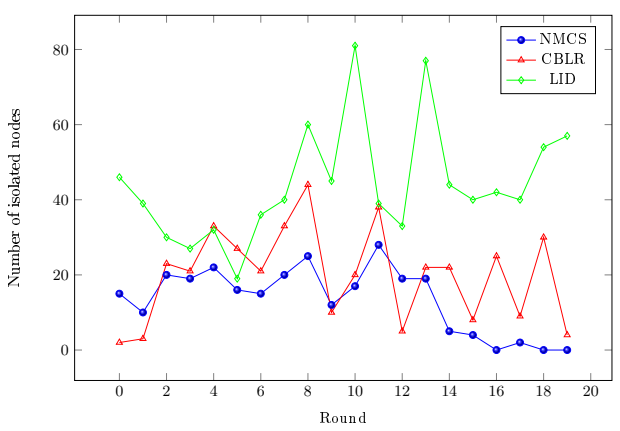

Fig. 4: Number of isolated nodes per round.

Actually, the clustering stability metrics, arrival/departure, and isolated nodes affect the performance of OTRB protocol. Table 2 shows that the more stable is the clustering protocol, the more OTRB performances are improved.

Figure 5 exhibits that the synchronization rate of OTRB using NMCS outperforms OTRB using the two other protocols. This is due to the improvement in the average of arrival/departure nodes which reveals successful clock synchronization process in the overall of nodes. Therefore, the number of messages generated in order to accomplish the synchronization process is minimized. On the other hand, the more the number of isolated nodes, the more supplementary synchronization requests are generated. For that, using NMCS under OTRB protocol improves the communication overhead compared to CBLR and LID protocols under OTRB (see Figure 6).

Table 2: Comparison.

\begin{tabular}{llcll}
\hline Implemented protocol & Arrival / departure nodes & Isolated nodes & Synchronization rate (s) & Communication overhead \\
\hline CBLR under OTRB & High & Low & Medium & High \\
LID under OTRB & Medium & Medium & Medium & High \\
NMCS under OTRB & Low & Medium & Improved & Improved \\
\hline
\end{tabular}




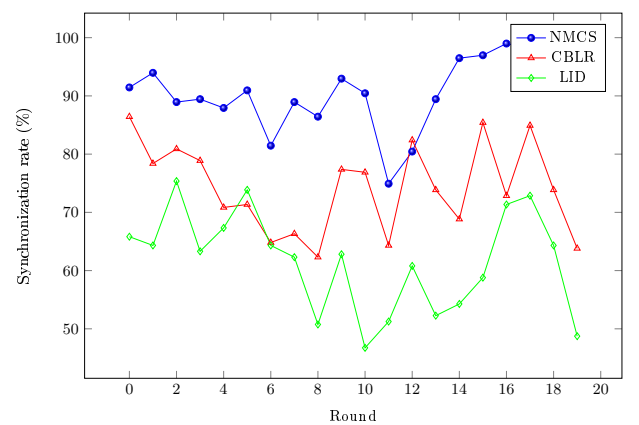

Fig. 5: Synchronization rate comparison.

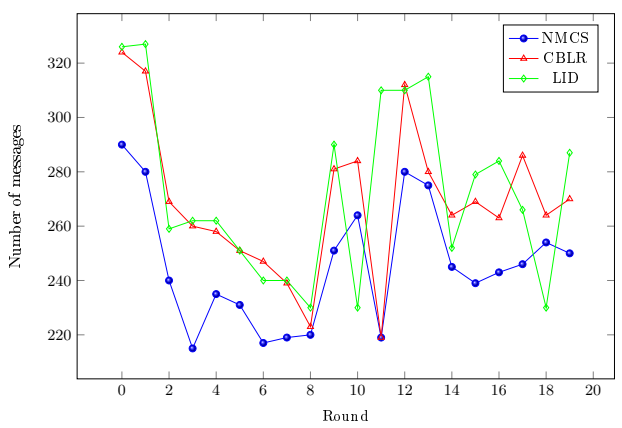

Fig. 6: Communication overhead comparison.

\section{Conclusion}

Because time synchronization is an essential attribute of VANET networks, because VANETs are included in a larger area: the IoV which itself is part of the largest networks: the IoT, then the quality of service of the synchronization process must fulfill the expected objective in accordance with network importance. The paper is dealing with the impact of the well know clustering nodes organization on time synchronization ORTB protocol in order to improve the latter capabilities. Several known clustering algorithms have been used by ORTB and the comparative analysis of simulation results reveals that better performance, in term of synchronization rate and average communication overhead, is offered by the high stability clustering algorithm. However, multi-metric algorithms can be used to further enhance the clustering stability and so the network performance. This can be the subject of future work.

\section{References}

1. Jijun Yin, Tamer ElBatt, Gavin Yeung, Bo Ryu, Stephen Habermas, Hariharan Krishnan, and Timothy Talty. Performance evaluation of safety applications over dsrc vehicular ad hoc networks. In Proceedings of the 1st ACM international workshop on Vehicular ad hoc networks, pages 1-9. ACM, 2004.

2. Khedidja Medani, Makhlouf Aliouat, and Zibouda Aliouat. Fault tolerant time synchronization using offsets table robust broadcasting protocol for vehicular ad hoc networks. AEU-International Journal of Electronics and Communications, 81:192-204, 2017.

3. Dahlia Sam, Cyrilraj Velanganni, and T Esther Evangelin. A vehicle control system using a time synchronized hybrid vanet to reduce road accidents caused by human error. Vehicular communications, 6:17-28, 2016.

4. Thore Graepel. Statistical physics of clustering algorithms. Technical Re-port, $171822,1998$.

5. Soumen Chakrabarti, Earl Cox, Eibe Frank, Ralf Hartmut Güting, Jiawei Han, Xia Jiang, Micheline Kamber, Sam S Lightstone, Thomas P Nadeau, Richard E Neapolitan, et al. Data mining: know it all. Morgan Kaufmann, 2008. 
6. Rasmeet S Bali, Neeraj Kumar, and Joel JPC Rodrigues. Clustering in vehicular ad hoc networks: taxonomy, challenges and solutions. Vehicular communications, $1(3): 134-152,2014$.

7. Craig Cooper, Daniel Franklin, Montserrat Ros, Farzad Safaei, and Mehran Abolhasan. A comparative survey of vanet clustering techniques. IEEE Communications Surveys \& Tutorials, 19(1):657-681, 2017.

8. Mario Gerla and Jack Tzu-Chieh Tsai. Multicluster, mobile, multimedia radio network. Wireless networks, 1(3):255-265, 1995.

9. Chunhung Richard Lin and Mario Gerla. Adaptive clustering for mobile wireless networks. IEEE Journal on Selected areas in Communications, 15(7):1265-1275, 1997.

10. Hang Su and Xi Zhang. Clustering-based multichannel mac protocols for qos provisionings over vehicular ad hoc networks. IEEE Transactions on Vehicular Technology, 56(6):3309-3323, 2007.

11. Yvonne Guenter, Bernhard Wiegel, and Hans Peter Großmann. Medium access concept for vanets based on clustering. In Vehicular Technology Conference, $200 \%$. VTC-2007 Fall. 2007 IEEE 66th, pages 2189-2193. IEEE, 2007.

12. RA Santos, RM Edwards, and NL Seed. Inter vehicular data exchange between fast moving road traffic using an ad-hoc cluster-based location routing algorithm and 802.11 b direct sequence spread spectrum radio. In PostGraduate Networking Conference, 2003.

13. Omer Kayis and Tankut Acarman. Clustering formation for inter-vehicle communication. In Intelligent Transportation Systems Conference, 200\%. ITSC $200 \%$. IEEE, pages 636-641. IEEE, 2007.

14. Zhigang Wang, Lichuan Liu, MengChu Zhou, and Nirwan Ansari. A position-based clustering technique for ad hoc intervehicle communication. IEEE Transactions on Systems, Man, and Cybernetics, Part C (Applications and Reviews), 38(2):201-208, 2008.

15. Hao Wu, Zhangdui Zhong, and Lajos Hanzo. A cluster-head selection and update algorithm for ad hoc networks. In Global Telecommunications Conference (GLOBECOM 2010), 2010 IEEE, pages 1-5. IEEE, 2010.

16. Khalid Abdel Hafeez, Lian Zhao, Zaiyi Liao, and Bobby Ngok-Wah Ma. A fuzzylogic-based cluster head selection algorithm in vanets. In Communications (ICC), 2012 IEEE International Conference on, pages 203-207. IEEE, 2012.

17. Zaydoun Y Rawashdeh and Syed Masud Mahmud. A novel algorithm to form stable clusters in vehicular ad hoc networks on highways. EURASIP Journal on Wireless Communications and Networking, 2012(1):15, 2012.

18. Efi Dror, Chen Avin, and Zvi Lotker. Fast randomized algorithm for 2-hops clustering in vehicular ad-hoc networks. Ad Hoc Networks, 11(7):2002-2015, 2013.

19. Behnam Hassanabadi, Christine Shea, L Zhang, and Shahrokh Valaee. Clustering in vehicular ad hoc networks using affinity propagation. Ad Hoc Networks, 13:535$548,2014$.

20. Jung-Hyok Kwon, Hyun Soo Chang, Taeshik Shon, Jai-Jin Jung, and Eui-Jik Kim. Neighbor stability-based vanet clustering for urban vehicular environments. The Journal of Supercomputing, 72(1):161-176, 2016.

21. Mengying Ren, Lyes Khoukhi, Houda Labiod, Jun Zhang, and Véronique Vèque. A mobility-based scheme for dynamic clustering in vehicular ad-hoc networks (vanets). Vehicular Communications, 2016.

22. Bharath Sundararaman, Ugo Buy, and Ajay D Kshemkalyani. Clock synchronization for wireless sensor networks: a survey. Ad hoc networks, 3(3):281-323, 2005. 